\title{
Confidence of others trumps confidence of self in social information use
}

\author{
Andrea Gradassi ${ }^{1}$, Wouter van den Bos ${ }^{1,2}$ \& Lucas Molleman ${ }^{1,3}$
}

\author{
${ }^{1}$ Department of Psychology, University of Amsterdam, The Netherlands \\ ${ }^{2}$ Center for Adaptive Rationality, Max Planck Institute for Human Development, Berlin, \\ Germany \\ ${ }^{3}$ Department of Social Psychology, Tilburg University, the Netherlands \\ Corresponding author: a.gradassi@uva.nl
}

\begin{abstract}
Humans live in a fundamentally social world. The behavior and judgments from friends, colleagues, television hosts and social media feeds drive our purchase decisions, induce lifestyle changes, and determine voting preferences. People tend to be more sensitive to social information when they are unsure about their own beliefs, and assign more weight to social information when its source is highly confident. However, little is known about the relative impact of the confidence of self and others on social information use, and how they jointly shape social transmission. Here we show with two incentivized decision-making experiments that the confidence of others had a substantially larger impact on social information use than people's own confidence. In tasks involving perceptual decisions (experiment 1; $\mathrm{N}=203$ ) and US election predictions (experiment 2; $\mathrm{N}=213$ ), participants could adjust their initial judgments upon observing judgments of others and were rewarded for accuracy. Adjustments were most strongly impacted by the confidence of others, particularly when participants' own confidence was low. Furthermore, confidence also affected adjustment heuristics: confident others prompted participants to compromise more often, rather than to stick with their initial judgments. Our results highlight how giving more weight on the confidence of others can be a double-edged sword: it can accelerate learning when confidence is related to accuracy, but it also leaves people vulnerable to sources who confidently share misinformation.
\end{abstract}

Keywords: Social Learning; Confidence; Social Learning Strategies; Social information use;

\section{Introduction}

Humans are constantly exposed to the behavior and opinions of others. Such social information can help individuals adjust to new social circumstances and improve the accuracy of their decisions (1). Social information is often accompanied by a certain degree of confidence: TV anchors and politicians take assertive postures, consultants are rarely unsure when offering advice, and social media feeds abound in opinionated comments. Research suggests that the impact of a statement critically depends on the 
confidence of its source (2-5). Confident witnesses are more likely to accurately identify suspects (6), and medical teams can efficiently converge on a correct diagnosis when an experienced doctor is confident about their assessment (7). However, when high confidence is unsupported by facts - as often in those holding dogmatic or extreme views $(8,9)$, misleading information may readily spread, exacerbating polarization, threatening the adoption of medical advice, and potentially swaying election outcomes $(10-12)$.

It is therefore crucial to understand when and how individuals use confidence (their own and that of others) to update their beliefs. Typically, people do not blindly follow others, but instead adaptively weigh social information against their own knowledge and beliefs (13-15). This weighting process can be aided by cues that speak directly to the value of social information, and people tend to prioritise sources with expertise, prestige, or success (16). However, in many real-word scenarios, individuals cannot observe cues about performance (of others nor themselves). In those cases, expressed or experienced confidence can serve as an indirect cue that guides how individuals weigh social information.

Here, confidence is defined as the subjective beliefs in the accuracy of an opinion or a judgment $(17,18)$. Individuals can access their confidence in their own judgments through introspection. Ample empirical evidence indicates that when unconfident, people tend to search more for social information, and rely more heavily on social information in making decisions (19-24). The reliability of social information may be gauged from the confidence expressed by its source, based on verbal or non-verbal expressions (e.g., stating "I'm sure"). Both confidence of self and others are associated with activity in the ventral medial prefrontal cortex (VMPFC), where they are integrated in the valuation of options (25-27). Although in everyday life, the confidence of self and others simultaneously shape social information use, experimental studies have hitherto only examined their effects in isolation $(2,3,5,19-24)$. It therefore remains unclear what is the relative impact of the confidence of self and others in integrating social information, and how the two interact to update individuals' beliefs.

We address these issues in three steps. First, we experimentally examine the relative impact of individuals' own confidence and that of others in integrating social information. Given that people generally prioritise their own information over that of others (i.e. egocentric discounting; (28)), one might expect that people will also focus mostly on their own confidence when confronted with social information. Alternatively, people might respond more strongly to the confidence of others than to their own confidence. Confidence cues are often prominent in everyday communication, and in those cases exposure to confident others may shift people's attention from their own (implicit) state of confidence to the (explicit) confidence of the other. Second, we investigate how the confidence of self and others interact. We expected the impact of others' confidence to be particularly pronounced when individuals' own confidence is low. Conversely, when confident, individuals should be more likely to simply ignore social information regardless of the confidence associated with it. Third, we delineate how the confidence of self and others influences individuals' heuristics of responding to social information, shaping the likelihood that they copy social information, stick with 
their own beliefs, or make a compromise between them. Understanding these heuristics is of crucial importance as they drive belief dynamics in groups and the spread of behaviors across social networks $(10,21,29)$.

We present the results of two pre-registered, incentivized experiments. Participants had to make judgments in an abstract estimation task (experiment 1) and in a naturalistic task in which they had to predict the results of the 2020 US elections (experiment 2). They could revise their estimates after observing the judgment and confidence of another participant, but did not receive any feedback throughout the task. The experiments are similar in structure, with the second task eliciting predictions about a current topic of global relevance (the 2020 US election), thus providing a more stringent test for validity of the effects of confidence on social influence.

The two experiments consistently show that the confidence of others had a substantially larger impact on social information use than the confidence of self. Furthermore, the effect of others' confidence was particularly pronounced when participants' own confidence was low, even though confidence and accuracy were not correlated in our experiment. Finally, when others' confidence was high, participants were less likely to stick with their own judgment and were more likely to compromise towards social information. Our study provides convergent evidence that, for good or ill, confident sources are heeded more, especially by those who are not confident themselves. We conclude that accounting for this bias can help individuals and groups make effective decisions, and prevent people from spreading misinformation by projecting high confidence.

\section{Results}

General setup. Both experiments involve decision making tasks in which participants made numerical estimates and were rewarded for accuracy. In experiment 1, they estimated the number of animals shown in an image (Fig. 1A). In experiment 2, they predicted the proportion of Democrats and Republican voters (out of 100) by state for the 2020 US presidential elections (Fig. 1B; Methods for details). After providing a first estimate and their confidence rating, participants observed the estimate of another participant ( $a$ 'peer') and the peer's confidence rating. Then, they provided a second estimate. We measure social information use $(s)$ as the relative adjustment from first to second estimates (see Fig. 1 for formal definition). 
A

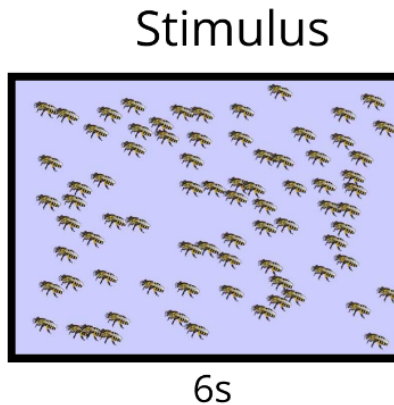

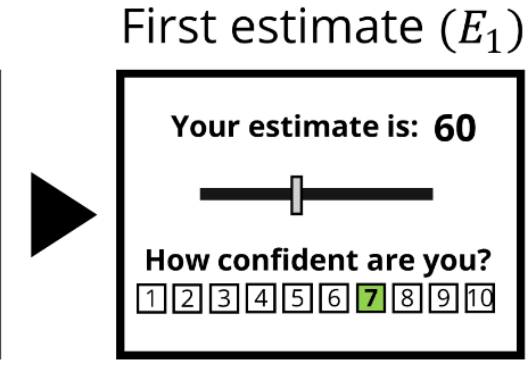

(up to) 30s

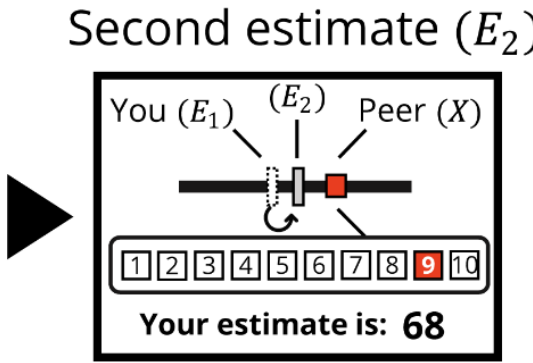

(up to) $30 \mathrm{~s}$

B

Stimulus \&

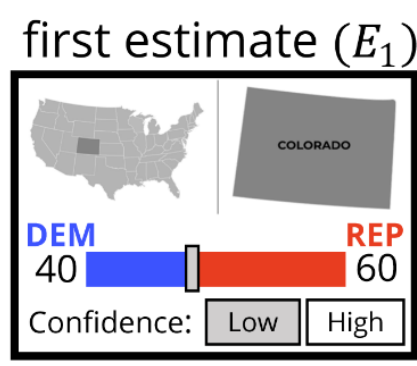

(up to) 30s
Second estimate $\left(E_{2}\right)$

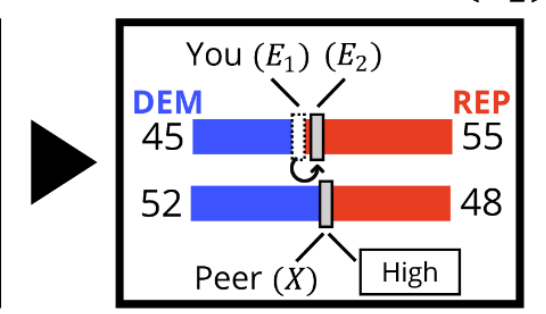

(up to) 30s

Fig. 1. Experimental Designs. Schematic representation of the task screens. A. A round of the estimation task (experiment 1). Participants first observe an image with animals on them for 6 seconds, provide their first estimate of their number ( $E_{1}$, grey rectangle) and rate their confidence in their estimate on a 1-10 scale. On the next screen, participants observe the estimate of a peer ( $X$, red square), the peer's confidence (highlighted on the scale), and their own first estimate ( $E_{1}$, white rectangle). Then they provide a second estimate ( $E_{2}$, grey rectangle). B. A round of the election prediction task (experiment 2). For a US state, participants predict the proportion of Democratic and Republican votes (out of 100; $E_{1}$ ), and rate their confidence as 'Low' or 'High'. On the next screen, they observe the prediction of a peer $(X)$ and their confidence, and make a second prediction $\left(E_{2}\right)$. In both experiments we measure social information use $(s)$ as the relative adjustment from first to second judgments $s=\frac{\left(E_{2}-E_{1}\right)}{\left(X-E_{1}\right)}$. When personal and social information are weighted equally, $s=0.5$. In addition to measuring adjustments in each round, we considered three heuristics: stay (ignore social information; $s=0$ ), compromise (move towards social information, $0<s<1$ ) and copy (adopt social information, $s=1)(19,23)$. For an overview of basic results, see SI §2.

To avoid deception, peer judgments presented to participants were selected from a previously collected pool of data in which different participants completed the same incentivised tasks without social information. In the main experiment, we selected social information to systematically vary the confidence of the observed peer, creating an equal number of rounds with low and high peer confidence (Methods). Combinations of 'Low' $(\mathrm{L})$ and 'High' $(\mathrm{H})$ confidence resulted in 4 experimental conditions - $\mathrm{LL}, \mathrm{LH}, \mathrm{HL}, \mathrm{HH}$ (where letters refer to own confidence and peer confidence, respectively). Our setup isolates the effects of confidence ratings on social information use, excluding effects of participants' knowledge of their peers' relative performance $(30,31)$, differential access to evidence (3), or interdependence of payoffs with others $(3,32)$. Furthermore, participants only received feedback about their accuracy after completing all trials of 
the task, to minimize any learning about their own performance and the possible value of social information.

Experiment 1. Participants completed 20 rounds of a validated judgment task (6) in which they were briefly shown images, and had to estimate the number of animals displayed in them (Fig. 1A). To manipulate participants' own confidence, the image was partly covered in half of the rounds (Methods). Confidence ratings (1-10) were not incentivised, and as pre-registered, they entered the analyses as Low if $<4$ and High if $>6$ (Methods).

Experiment 2. Participants completed 20 rounds of a novel, naturalistic prediction task (Fig. 1B). In each round, they were asked to predict the outcome of the 2020 US presidential election for one state (see Table S1 for full list). Confidence ratings ('Low' or 'High') were incentivized by telling participants that rounds in which they expressed high confidence would be twice more likely to be selected for payment than rounds with low confidence.

People prioritize personal over social information. Overall, participants revised their judgments in the direction of social information by .37 in experiment 1, and by .30 in experiment 2 . This magnitude of adjustments is comparable to studies using similar designs (first estimate, then revise; $(13,15,33)$ ). In both experiments, participants' average adjustments rarely exceeded .50 . This result is indicative of the widely documented phenomenon of egocentric discounting, in which people assign more weight to their own beliefs than to social information $(28,34)$.

Social information use is more strongly impacted by peer confidence than by own confidence. In both experiments, adjustments varied substantially with experimental conditions (Fig. 2B-C). The magnitude of adjustments decreased when participants' own confidence was high (experiment $1: \mathrm{b}=-.05,95 \%$ C.I. $=(-.08,-.02)$; experiment $2: \mathrm{b}=-.03$, $95 \%$ C.I. $=(-.04,-.00))$, and increased when peer confidence was high (experiment $1: \mathrm{b}=$ $.12,95 \%$ C.I. $=(.09, .15)$; experiment $2: \mathrm{b}=.08,95 \%$ C.I. $=(.06, .10)$; Fig. 2A). Notably, the effect of peer confidence was about twice as large as the effect of participants' own confidence. This suggests that people' s social information use is influenced more by others' confidence than by their own confidence. 

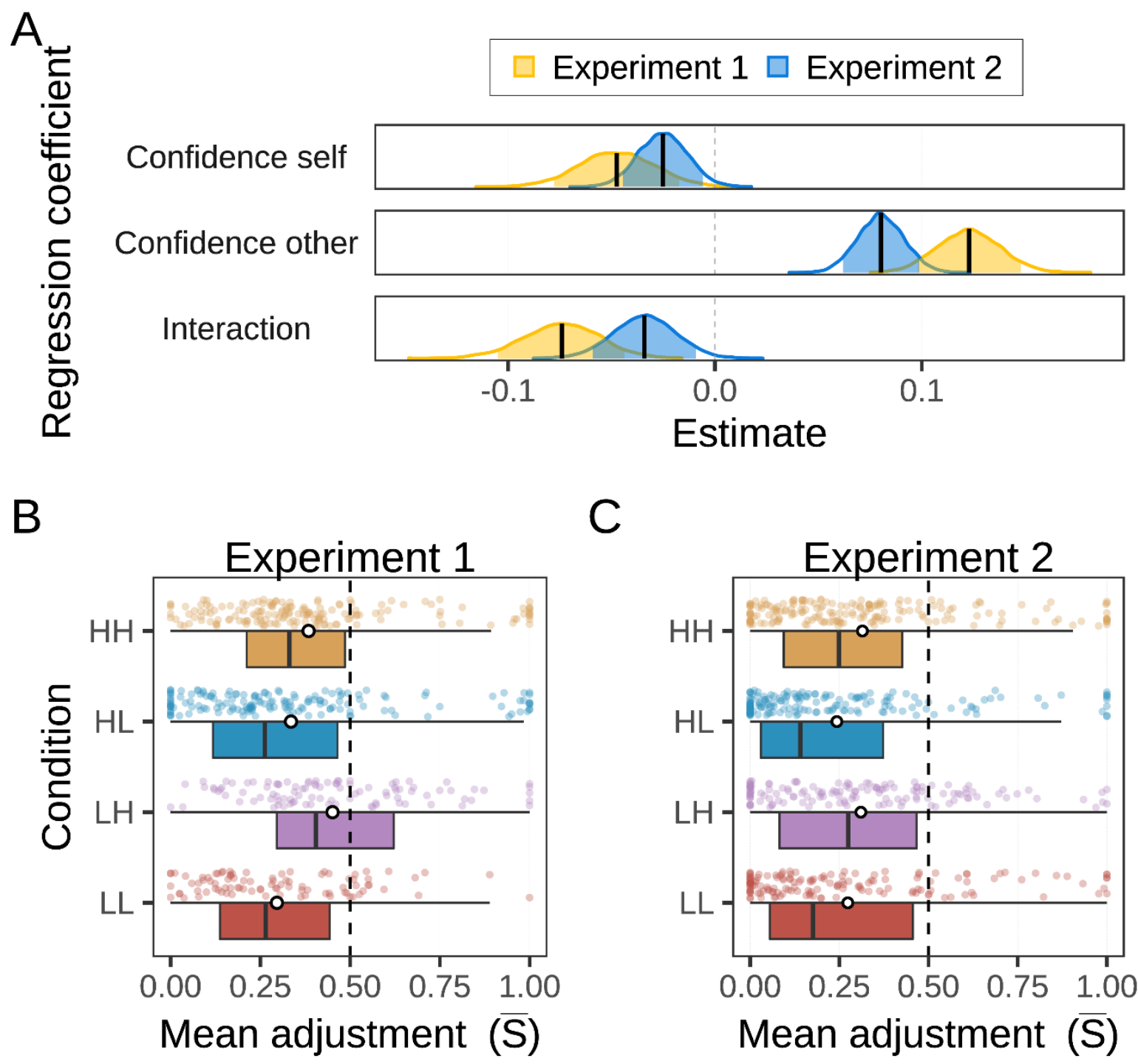

Fig. 2. Confidence and social information use. A. Posterior distributions of regression coefficients estimated with a Bayesian Linear Mixed Model with 'participant' as random intercept (see Table S2 \& S3 for full model specification and robustness checks). Yellow and blue distributions represent the estimated coefficients for experiment 1 and 2, respectively. Black vertical lines indicate the mean of the distributions. The highlighted areas under the curves indicate $95 \%$ posterior credible intervals. B-C. Mean adjustments $(\bar{S})$ by experimental condition for both experiments. Each dot represents the mean value of $s$ for one participant. The first and second letters of the $y$-axis labels indicate the confidence of the participant and the observed peer, respectively ( $\mathrm{L}=\mathrm{Low}, \mathrm{H}=\mathrm{High}$ ). Black vertical bars in the boxplots show medians, white dots show means. Dashed black vertical lines indicate the point of equal weighting of personal and social information $(\bar{S}=0.5)$. Values of $\bar{S}$ were mostly below 0.5 , even in the LH condition (purple dots). For further details on adjustments in individual rounds, see Fig. 3.

Individuals respond the most to peer confidence when their confidence is low. Participants' own confidence and the confidence of the peer credibly interacted with each other (experiment 1: b $=-.07$, C.I. $=(-.11,-.04)$; experiment $2: \mathrm{b}=-.03,95 \%$ C.I. $=(-$ $.06,-.00)$; Fig. 2A). In both experiments, the impact of peer confidence on social information was most pronounced when participants' own confidence was low. This 
indicates that the internal state of individuals - i.e., being more or less confident - may suppress or enhance responses to peer confidence in integrating social information.

Peer confidence drives the use of heuristics. Figure 3 shows the distribution of heuristics participants used in individual rounds, broken down by experiment and conditions. Overall, 'compromise' was most frequent (experiment 1: 72\%, experiment 2 $60 \%$ of cases), followed by 'stay' (19\% and $30 \%)$, while 'copy' was rare (9\%, and $8 \%$, with most instances of 'copy' belonging to a small subgroup, see figure S1). Across conditions, the majority of compromising cases were ( $0<s<0.5$; Fig. 3, light green), implying that participants discounted social information relative to their own estimate. Notably, the largest proportion of rounds in which is either $s=.5$ or $s>.5$ is observed in the LH condition. The relative frequency of the adjustment strategies markedly varied by condition. In both experiments, 'stay' was credibly more likely in the $\mathrm{LL}, \mathrm{HL}$ and $\mathrm{HH}$ conditions than in the LH condition. Compromising followed a complementary pattern: participants were equally likely to compromise in the $\mathrm{LL}, \mathrm{HL}$ and $\mathrm{HH}$ conditions, and credibly more likely to do so in the LH condition. The frequency of 'copy' did not credibly vary between conditions (see Table S4 \& S5 for the detailed statistics). Overall, these results indicate that people are most likely to change their mind and make a compromise when their own confidence is low and the confidence of their social source is high.
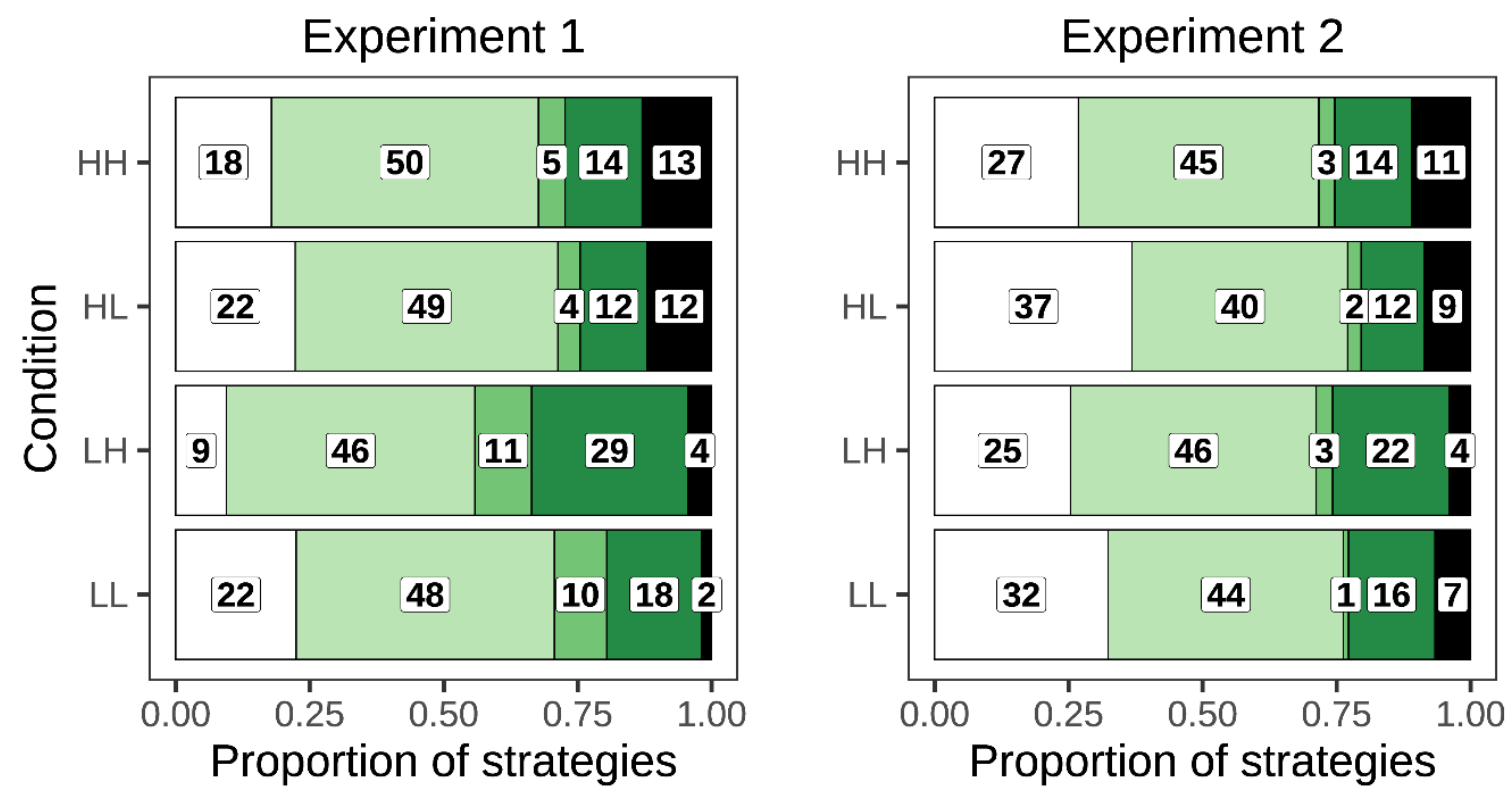

Adjustment strategy

$\square$ Stay $\square$ Comp [s<0.5] $\square$ Comp [s=0.5] $\square$ Comp [s>0.5] $\square$ Copy

Fig. 3. Frequencies of heuristic strategies. Bars show the relative frequencies of staying, compromising and copying, broken down by experimental condition. Numbers in white boxes indicate the percentage of cases in each bin. Instances of 'compromise' (green bars) are further broken down into cases where participants gave more weight to personal information ( $<<.5$, light green), equal weight to personal and social information ( $s=.5$, intermediate green), or more 
weight to social information ( $\mathrm{s}>.5$; dark green). For an overview of the strategies broken down by participant, see Fig. S1.

\section{Discussion}

In this paper we examined how social information use is shaped by the concerted action of individuals' own confidence and the confidence of others. Our experiments provide convergent behavioral evidence for three phenomena. First, social information use is impacted more by others' confidence than by their own confidence. Second, the effect of others' confidence is particularly strong when people's own confidence is low. Third, highly confident others are more likely to induce individuals to compromise.

In both our experiments, and consistent with previous studies $(21,28,33,34)$, participants assigned more weight to their own estimates than to those of others (mean $s<0.5$; Fig. 2A,B). It is therefore surprising that social information use was impacted more strongly by the confidence of others than by their own. This result might stem from the fact that - as in many real-life situations - peer confidence was salient and explicitly communicated, while participants' own confidence was only implicitly available. Given that salient features tend to be prioritised in decision-making $(35,36)$, the confidence of others might have particularly strong effects on social information use. Those seeking to increase their influence can take advantage of this asymmetry by strategically projecting high confidence. Such behavior can be particularly dangerous in online environments, where individuals typically take little time to process information and often use contextual cues (e.g. number of likes, retweets) to evaluate its quality (37) while confidence is not always a reliable cue. Raising awareness about saliency effects can help individuals effectively weigh the value of information. In practice, this means 1) using confidence cues only when they reliably predict accuracy $(5,38)$, and 2 ) counter the saliency effect by shifting attention from confidence of others to own confidence.

Although participants' confidence in their own judgments had relatively modest effects on social information use, it did modulate the impact of the confidence of others (Fig. 2C). That is, when participants were confident, they tended to make their second judgments with little regard to the judgments of others, and little sensitivity to others' confidence. This might reflect participants being 'cognitive misers' (39) saving effort by ignoring social information when it is not needed. This behavior is consistent with observations that high confidence is associated with reduced search for social information (40). Our result shows that confidence-based social transmission depends on the interplay between confidence of learners and confidence of sources, and suggests that individuals' own uncertainty reflects their willingness to invest effort to acquire or process social information.

Confidence of others, but not their own confidence, produced pronounced effects on heuristics (Fig. 3). When other's confidence was high, people were less likely to stay with their initial beliefs and more willing to compromise between their beliefs and social information. Adjusting towards the opinion of confident others can improve group 
decisions (41), but it will do so only if confidence reliably predicts accuracy (42). Our results suggest that, in the absence of feedback about individual performance, people maintain the default assumption that others' confidence is well calibrated to their accuracy. In addition, when groups have to solve problems that require coordination (e.g. designing policies to curb carbon emissions or to reduce covid-19 infections), efficiently reaching an agreement becomes key. In these scenarios, compromising can foster consensus-building (29), and our results suggest that confident individuals may accelerate this process by prompting their peers to change their minds.

Our work is not without limitations. First, during the experiment, participants did not receive any feedback on their own performance or that of others. They could therefore not learn how accurate their estimates were. This mimics decision situations that usually lack objective information about the accuracy of beliefs, e.g., in courtrooms (43), on stock exchanges $(4,44)$, or on social media (45). However, future studies may assess if the observed disproportionate influence of confident peers holds when people can learn how good they are at a given task (e.g., through recognition by colleagues or using feedback from fact checking sources (e.g. https://www.factcheck.org/,

https://www.politifact.com/)). If people had evidence that they perform better than their peers, they may use less social information - or ignore it altogether. Second, it would be of theoretical interest to compare the impact of confidence cues to other cues that shape social information use $(16,46)$. For example, in a recent study where people could choose amongst different types of social cues, they mostly opted for decisions of experts over decisions of prestigious peers (47). It remains an open question whether people would pay less attention to others' confidence when they could learn about their success or social status (16). Finally, social information use might be shaped by the interaction of confidence with other social cues, like expertise. For example, would unconfident experts be followed less than confident laymen?

Our findings contribute to literature on social learning by extending the research on how people weigh social information. Specifically, we find that while one's own confidence impacts social transmission, people value the confidence of others more strongly, taking it as a reliable signal. Our findings highlight two important considerations for everyday decision-making. First, the implicit belief in the validity of confidence signals may be justified in cooperative environments, where learners and sources share common goals (e.g., a hiring committee trying to select the best applicant), but leaves individuals vulnerable to those who falsely project confidence (e.g. social media influencers trying to sell sponsored products; $(3,32,48)$. Second, effective use of confidence as a signal of reliability assumes that our own, but also other's confidence is well calibrated and communicated. This is especially important for the communication of information on complex issues, such as climate change, where experts may be more reluctant to express full confidence on a given solution whereas those with a superficial understanding may be convinced of their positions (49). Thus, when individuals are uncertain and are tempted to use social information from confident others, they should carefully consider their source's interests (37), and factcheck the validity of their source's claims. 


\section{Materials and Methods}

General procedures. Both experimental designs were pre-registered on the Open Science Framework (experiment 1: https://osf.io/wbpd7/; experiment 2: https://osf.io/ar4kx/ ). Deviations from the pre-registered analysis can be found in the Supplementary Text. Participants were recruited online through Amazon Mechanical Turk. Participants were selected only amongst USA residents with a history of at least 95\% approved tasks. Procedures were approved by the local Ethics Review Board (case number 2020-DP12605).

Analyses. All the analyses were performed using the statistical software $R$ (R Core Team, 2021), in the IDE RStudio (RStudio Team, 2021). Data cleaning and wrangling were supported by the tidyverse package (50), while Bayesian regression models were fitted using brms (51). As pre-registered, and consistent with previous studies using similar paradigms $(15,29)$, statistical models fitted to $s$ only included rounds in which second judgments were a weighted average of own first judgments and social information (that is, $0 \leq s \leq 1$ ), which were the large majority of rounds (95\% in experiment 1, 90\% in experiment 2).

\section{Experiment 1}

Participants. 203 participants (mean age $=37.87$, s. $d=10.85$, range $=22-74,34.5 \%$ female) were recruited for study 1 . They were compensated with a fixed fee of $\$ 2.00$, plus an additional bonus up to $\$ 1.00$ (average $=\$ 0.52, \mathrm{SD}=\$ 0.20$ ) depending on their performance, for a total expected payment of $\sim \$ 2.50(\sim 10.00 / \mathrm{hr})$. The bonus was calculated by randomly selecting one of the 20 rounds, and evaluating participants' performance for that specific round (for each animal their estimate was off, we subtracted $\$ 0.05$ from their bonus of $\$ 1.00$, the bonus could not become negative). This setup incentivized participants to invest an equal amount of effort in each round and avoided income effects.

Task Design. The experiment consisted of a short (15 minutes) decision-making task (Fig. 1A). In each of the 20 rounds, participants saw for 6 seconds an image containing a number of animals (between 50 and 100). The image was either fully revealed (complete information condition) or partially revealed (uncertain condition). The aim of this first manipulation was to vary participants' baseline subjective confidence in their own estimate. After the image had disappeared, participants were asked to give an estimate of the correct number of animals by using a slider, and to rate their confidence in their estimate on a scale from 1 ('Not confident at all') to 10 ('Extremely confident').

Participants subsequently observed the estimate of a previous participant (a 'peer'), who saw the same stimulus for the same amount of time, and had the same task of estimating the number of animals on it. On the same screen, participants observed the peer's confidence rating, expressed on the same scale as the one that participants used.

This information (estimate and confidence rating) was collected in an earlier iteration of the experiment, where $\mathrm{N}=62$ participants played the solo condition and after that 
reported their confidence ratings. Thus, all the social information displayed was real. From this pool of pre-recorded data, we selected in each round the estimate of a peer that was closest to the target value $X^{\prime}$. We defined $X^{\prime}$ such that it always pointed in the direction of the true value. Specifically, we defined $X^{\prime}=E_{1} \cdot(1+/-\Delta)$, where $E_{1}$ is the participants initial estimate, where $\Delta=0.2$ if $E_{1}$ was lower than the true value, and $\Delta=-0.2$, if $E_{1}$ was higher than the true value (15). If $E_{1}$ was equal to the true value, either value of $\Delta$ was chosen with equal probability. $\Delta$ was selected to leave participants enough room to adjust and controlling for distance effects that might influence adjustments $(29,33)$.

For a peer estimate to be selected as social information, we applied one additional criterion: it had to be associated with a confidence rating that matched the experimental treatment of the current round. The ratings were distributed on a scale from 1 (Not confident at all) to 10 (Extremely confident). Three experimental treatments were derived from these ratings: low peer confidence (ratings between 1 and 4), medium peer confidence (ratings between 5 and 6 ) and high peer confidence (ratings between 7 and 10). These cut-off points were chosen to create a separation between the two treatments of interest, namely low peer confidence and high peer confidence (see preregistered design).

Over the 20 rounds, participants saw social information reflecting low confidence (between 1 and 4) in 8 rounds, social information reflecting high confidence (between 7 and 10) in 8 rounds, and social information reflecting medium confidence (between 5 and 6 ) in 4 rounds. We used the medium confidence rounds as 'filler' rounds, i.e. rounds that made the experiment more realistic from the participants' point of view, by including more values from the confidence scale, but which were excluded from some of the analyses. $\Delta$ was kept constant at 0.2 in 'filler' rounds as well. For this reason, we included fewer medium confidence rounds.

In experiment 1 , as pre-registered, confidence ratings of peers were grouped by categories (Low: 1-4; Medium: 5-6; High: 7-10 on a 10-point scale), and filler rounds in which confidence was 'Medium' were excluded from the analysis (alternative analyses in which confidence is treated as continuous variable produce qualitatively identical results; see Table S6).

\section{Experiment 2}

Participants. 213 participants (mean age $=38.84, \mathrm{~s} . \mathrm{d}=11.70$, range $=20-71,45.54 \%$ female) were recruited for study 2. Data was collected during the week prior to the 2020 US presidential election (October 26th-29th 2020). Compensation consisted of a fixed fee of $\$ 1.70$, plus an additional bonus up to $\$ 2.00$ (average $=\$ 0.94, \mathrm{SD}=\$ 0.60$ ) depending on the performance, for a total expected payment of $\sim \$ 2.70(\sim \$ 12.00 / \mathrm{hr})$. The bonus was calculated by randomly selecting two out of the 20 rounds, and evaluating participants' predictions for those rounds. For each selected round, for each vote their prediction was off, we subtracted $\$ 0.05$ from their bonus; the bonus for a round could not become negative. In this way, we incentivized participants to invest an 
equal amount of effort in each round and avoided income effects. Bonuses were paid out once the final election results could be estimated with a $1 \%$ margin of error.

Task design. The experiment consisted in a short (15 - 20 minutes) prediction task. Participants had to predict the election outcome for a sample of 20 USA states (see Table S1 for a complete list). Participants played 20 rounds, and in each they had to predict the percentage of votes for Democrats and Republican for one State. The question was phrased as follows: "out of 100 voters from [state name], how many will vote Democrat or Republican?" First, participants completed a 'solo condition', in which they were prompted to provide a first prediction by using a slider, and rated their confidence in it by clicking on one of two buttons, 'low' or 'high'. Participants then moved to the social information part of the experiment, where they could see: 1) the prediction of a previous participant in the experiment for that same state 2) the confidence rating of the same peer for that round ('low' or 'high'; Fig. 1B).

This information (estimate and confidence rating) was drawn from a previous sample of $\mathrm{N}=100$ participants who completed the same task without social information. As in experiment 1 , the target social information $X^{\prime}$ was defined $X^{\prime}=E_{1} \cdot(1+/-\Delta)$, where $E_{1}$ is the participants initial estimate. Differently from experiment $1, \Delta$ was not constant, but ranged between $.10-19$. Such range still gave participants enough room to adjust, and allowed to preserve a realistic and varied range of stimuli. The final distribution of target values (e.g. how many rounds had a target at 10 pp., 12.pp away, etc.) was identical for each participant. Finally, to enhance believability, in the two remaining (filler) rounds, social information was either very close or very far from the participant's first prediction.

\section{Data and code sharing plan}

Data and code will made accessible through a public repository upon manuscript publication.

\section{Acknowledgments}

The research was supported by the following research grants: Amsterdam Brain and Cognition grant 2018 (LM); Dutch Organization for Scientific Research grant NWO-427 VIDI 016.Vidi.185.068 (WB); European Research Council grant ERC-2018-StG-803338 (WB). We thank Mubashir Sultan for designing the stimuli and the help piloting the behavioral task for experiment 2, Dominik Deffner for the discussion on how to improve the analysis of the data and all the members of the Connected Minds lab for the providing feedback at various step of this study.

Author Contributions: A.G, L.M. and W.v.d.B. designed the study. A.G and L.M. programmed the experiments, A.G. collected the data. All authors were involved in designing the analysis plan, interpreting the results, and writing and editing the manuscript.

Competing Interest: The authors declare no conflicts of interest. 


\section{References}

1. L. Rendell, et al., Why Copy Others? Insights from the Social Learning Strategies Tournament. Science 328, 208-213 (2010).

2. P. Zarnoth, J. A. Sniezek, The Social Influence of Confidence in Group Decision Making. Journal of Experimental Social Psychology 33, 345-366 (1997).

3. B. D. Pulford, A. M. Colman, E. K. Buabang, E. M. Krockow, The persuasive power of knowledge: Testing the confidence heuristic. Journal of Experimental Psychology: General 147, 1431 (20180823).

4. P. C. Price, E. R. Stone, Intuitive evaluation of likelihood judgment producers: evidence for a confidence heuristic. Journal of Behavioral Decision Making 17, 39-57 (2004).

5. D. Bang, et al., Confidence matching in group decision-making. Nature Human Behaviour (2017) https:/doi.org/10.1038/s41562-017-0117.

6. J. T. Wixted, G. L. Wells, The Relationship Between Eyewitness Confidence and Identification Accuracy: A New Synthesis. Psychol Sci Public Interest 18, 10-65 (2017).

7. R. H. J. M. Kurvers, et al., Boosting medical diagnostics by pooling independent judgments. PNAS 113, 8777-8782 (2016).

8. M. Rollwage, R. J. Dolan, S. M. Fleming, Metacognitive Failure as a Feature of Those Holding Radical Beliefs. Current Biology 28, 4014-4021.e8 (2018).

9. J.-W. van Prooijen, A. P. M. Krouwel, Psychological Features of Extreme Political Ideologies. Curr Dir Psychol Sci 28, 159-163 (2019).

10. D. Centola, The Spread of Behavior in an Online Social Network Experiment. Science 329, 1194-1197 (2010).

11. E. Bakshy, I. Rosenn, C. Marlow, L. Adamic, The role of social networks in information diffusion in Proceedings of the 21st International Conference on World Wide Web, WWW '12., (Association for Computing Machinery, 2012), pp. 519-528.

12. R. H. J. M. Kurvers, et al., Strategic disinformation outperforms honesty in competition for social influence. iScience 24, 103505 (2021).

13. I. Yaniv, Receiving other people's advice: Influence and benefit. Organizational Behavior and Human Decision Processes 93, 1-13 (2004).

14. S. Bonaccio, R. S. Dalal, Advice taking and decision-making: An integrative literature review, and implications for the organizational sciences. Organizational Behavior and Human Decision Processes 101, 127-151 (2006).

15. L. Molleman, R. H. J. M. Kurvers, W. van den Bos, Unleashing the BEAST: a brief measure of human social information use. Evolution and Human Behavior 40, 492-499 (2019).

16. R. L. Kendal, et al., Social learning strategies: Bridge-building between fields. Trends in cognitive sciences 22, 651-665 (2018). 
17. A. Pouget, J. Drugowitsch, A. Kepecs, Confidence and certainty: distinct probabilistic quantities for different goals. Nat Neurosci 19, 366-374 (2016).

18. D. K. Peterson, G. F. Pitz, Confidence, uncertainty, and the use of information. Journal of Experimental Psychology: Learning, Memory, and Cognition 14, 85-92 (1988).

19. T. J. H. Morgan, L. E. Rendell, M. Ehn, W. Hoppitt, K. N. Laland, The evolutionary basis of human social learning. Proceedings of the Royal Society B: Biological Sciences 279, 653-662 (2012).

20. J. A. Minson, J. S. Mueller, The Cost of Collaboration: Why Joint Decision Making Exacerbates Rejection of Outside Information. Psychol Sci 23, 219-224 (2012).

21. M. Moussaïd, J. E. Kämmer, P. P. Analytis, H. Neth, Social influence and the collective dynamics of opinion formation. PloS one 8, e78433 (2013).

22. K. Desender, A. Boldt, N. Yeung, Subjective Confidence Predicts Information Seeking in Decision Making. Psychol Sci 29, 761-778 (2018).

23. N. Pescetelli, N. Yeung, The role of decision confidence in advice-taking and trust formation. Journal of Experimental Psychology: General 150, 507 (20201001).

24. F. Gino, A. W. Brooks, M. E. Schweitzer, Anxiety, advice, and the ability to discern: Feeling anxious motivates individuals to seek and use advice. Journal of Personality and Social Psychology 102, 497-512 (2012).

25. M. Lebreton, R. Abitbol, J. Daunizeau, M. Pessiglione, Automatic integration of confidence in the brain valuation signal. Nat Neurosci 18, 1159-1167 (2015).

26. D. Campbell-Meiklejohn, A. Simonsen, C. D. Frith, N. D. Daw, Independent Neural Computation of Value from Other People's Confidence. J. Neurosci. 37, 673-684 (2017).

27. S. Gherman, M. G. Philiastides, Human VMPFC encodes early signatures of confidence in perceptual decisions. eLife 7, e38293 (2018).

28. O. Morin, P. O. Jacquet, K. Vaesen, A. Acerbi, Social information use and social information waste. Philosophical Transactions of the Royal Society B: Biological Sciences 376, 20200052 (2021).

29. L. Molleman, et al., Strategies for integrating disparate social information. Proceedings of the Royal Society B: Biological Sciences 287, 20202413 (2020).

30. J. A. Sniezek, T. Buckley, Cueing and cognitive conflict in Judge-Advisor decision making. Organizational Behavior and Human Decision Processes 62, 159-174 (1995).

31. J. A. Sniezek, L. M. Van Swol, Trust, Confidence, and Expertise in a Judge-Advisor System. Organizational Behavior and Human Decision Processes 84, 288-307 (2001).

32. J. P. Thomas, R. G. McFadyen, The confidence heuristic: A game-theoretic analysis. Journal of Economic Psychology 16, 97-113 (1995).

33. B. Jayles, et al., How social information can improve estimation accuracy in human groups. PNAS 114, 12620-12625 (2017). 
34. I. Yaniv, E. Kleinberger, Advice Taking in Decision Making: Egocentric Discounting and Reputation Formation. Organizational Behavior and Human Decision Processes 83, 260-281 (2000).

35. K. Jung, J. Jeong, J. D. Kralik, A Computational Model of Attention Control in MultiAttribute, Context-Dependent Decision Making. Frontiers in Computational Neuroscience 13, 40 (2019).

36. I. Krajbich, Accounting for attention in sequential sampling models of decision making. Current Opinion in Psychology 29, 6-11 (2019).

37. A. Kozyreva, S. Lewandowsky, R. Hertwig, Citizens Versus the Internet: Confronting Digital Challenges With Cognitive Tools. Psychol Sci Public Interest 21, 103-156 (2020).

38. G. Pennycook, et al., Shifting attention to accuracy can reduce misinformation online. Nature 592, 590-595 (2021).

39. S. T. Fiske, S. E. Taylor, Social cognition, 2nd ed (Mcgraw-Hill Book Company, 1991).

40. N. Pescetelli, A.-K. Hauperich, N. Yeung, Confidence, advice seeking and changes of mind in decision making. Cognition 215, 104810 (2021).

41. B. Bahrami, et al., Optimally Interacting Minds. Science 329, 1081-1085 (2010).

42. A. Koriat, When Are Two Heads Better than One and Why? Science 336, 360-362 (2012).

43. R. Cramer, S. Brodsky, J. DeCoster, Expert Witness Confidence and Juror Personality: Their Impact on Credibility and Persuasion in the Courtroom. The journal of the American Academy of Psychiatry and the Law 37, 63-74 (2009).

44. D. Egan, C. Merkle, M. Weber, Second-order beliefs and the individual investor. Journal of Economic Behavior \& Organization 107, 652-666 (2014).

45. H. Allcott, M. Gentzkow, Social Media and Fake News in the 2016 Election. Journal of Economic Perspectives 31, 211-236 (2017).

46. R. McElreath, et al., Applying evolutionary models to the laboratory study of social learning. Evolution and Human Behavior 26, 483-508 (2005).

47. C. Brand, S. Heap, T. Morgan, A. Mesoudi, the emergence and adaptive use of prestige in an online social learning task. Scientific reports 10, 1-11 (2020).

48. , Simple heuristics in a social world (Oxford University Press, 2013).

49. G. Pennycook, R. M. Ross, D. J. Koehler, J. A. Fugelsang, Dunning-Kruger effects in reasoning: Theoretical implications of the failure to recognize incompetence. Psychon Bull Rev 24, 1774-1784 (2017).

50. H. Wickham, et al., Welcome to the Tidyverse. Journal of Open Source Software 4, 1686 (2019).

51. P.-C. Bürkner, brms: An R Package for Bayesian Multilevel Models Using Stan. Journal of Statistical Software 80, 1-28 (2017). 


\section{Supplementary Information}

\section{Supplementary Information Text}

\section{\$1 Deviation from preregistration}

\section{Experiment 1}

The experiment reported here, was conceived after collecting and analyzing the data from 3 previous experiments as presented in the pre-registration (see: https://osf.io/wbpd7/).

In these previous experiments, the effects of confidence of self and others were analyzed separately, next to the effects of stimulus uncertainty.

In all three studies, we observed both effects of confidence self and others, but no consistent effects of stimulus uncertainty. For this reason, in the final experiment reported here, which was the final confirmatory study, we only tested for effects of confidence, and not of stimulus uncertainty.

\section{Experiment 2}

In the pre-registered analysis (https://osf.io/ar4kx/), we included additional control variables for robustness checks (cf. Analyses, Model 2). Including those variables in the regression models did not change the main results (see Table S2). In the main text we only report the results relevant to the comparison to experiment 1.

Additionally, in the pre-registered model, confidence of self and others were included as main effects, and not as interactions. This choice was made to make the interpretation of the models with the multiple control variables simpler. However, in the main text we only focused on the role of confidence, and we thus can interpret the more fine-grained model (i.e. including the interaction) more precisely. 


\section{$\S 2$ Basic behavioral results.}

Experiment 1. On aggregate, participants underestimated the number of animals by $12 \%$, as indicated by the intercept of a linear model fitted to $\frac{\text { first estimate }}{\text { nanimals }}\left(\beta_{0}=.88\right.$, S.E. $\left.=0.01\right)$, in line with other studies using estimation tasks unfamiliar to the participants (Molleman et al., 2019, 2020).

Confidence ratings. On average, participants' confidence ratings were slightly above the midpoint of the confidence scale (mean confidence $=6.20, \mathrm{~s} . \mathrm{d}=2.35$, range 1-10).

Confidence and accuracy. Participants' predictions were not significantly more accurate in rounds where they expressed higher confidence, indicating that confidence ratings were not reflective of better estimation abilities (linear mixed model, $b=.03, t=1.28, p=.20$ ).

Experiment 2. Participants' initial predictions of the election outcomes were remarkably accurate: on average participants overestimated the share of votes for the Democratic party by $6 \%$, as indicated by the intercept of a linear model fitted to $\frac{\text { prediction }}{\text { election outcome }}\left(\beta_{0}=1.06\right.$, S.E. $=0.01$; Fig. S2 for a breakdown of prediction by State). This indicates that participants took their job seriously, and compared to the $12 \%$ underestimation observed in experiment 1 , suggests that the task was slightly easier. Confidence ratings. Participants reported "Low" confidence ratings on $44 \%$ of the rounds, and "High" confidence in the remaining 56\%, indicating an even split. Confidence and accuracy. As in task 1, participants' predictions were not significantly more accurate in rounds where they expressed higher confidence, indicating that confidence ratings were not reflective of better knowledge (linear mixed model, $b=.01, t=.79, p=.43$ ).

Since predictions and confidence reports might have been affected by participants' previous knowledge or expectations, we tested our hypotheses in a model which also accounted for four additional pre-registered predictors: (i) participants self-reported expertise in politics, (ii) preference for the observed outcome, (iii) whether participant and social source predicted the same winner, and (iv) state population size. Including these additional predictors in the model did not affect the main findings on confidence (Table S3). 
Fig. S1. Proportion of strategies use by individual participants
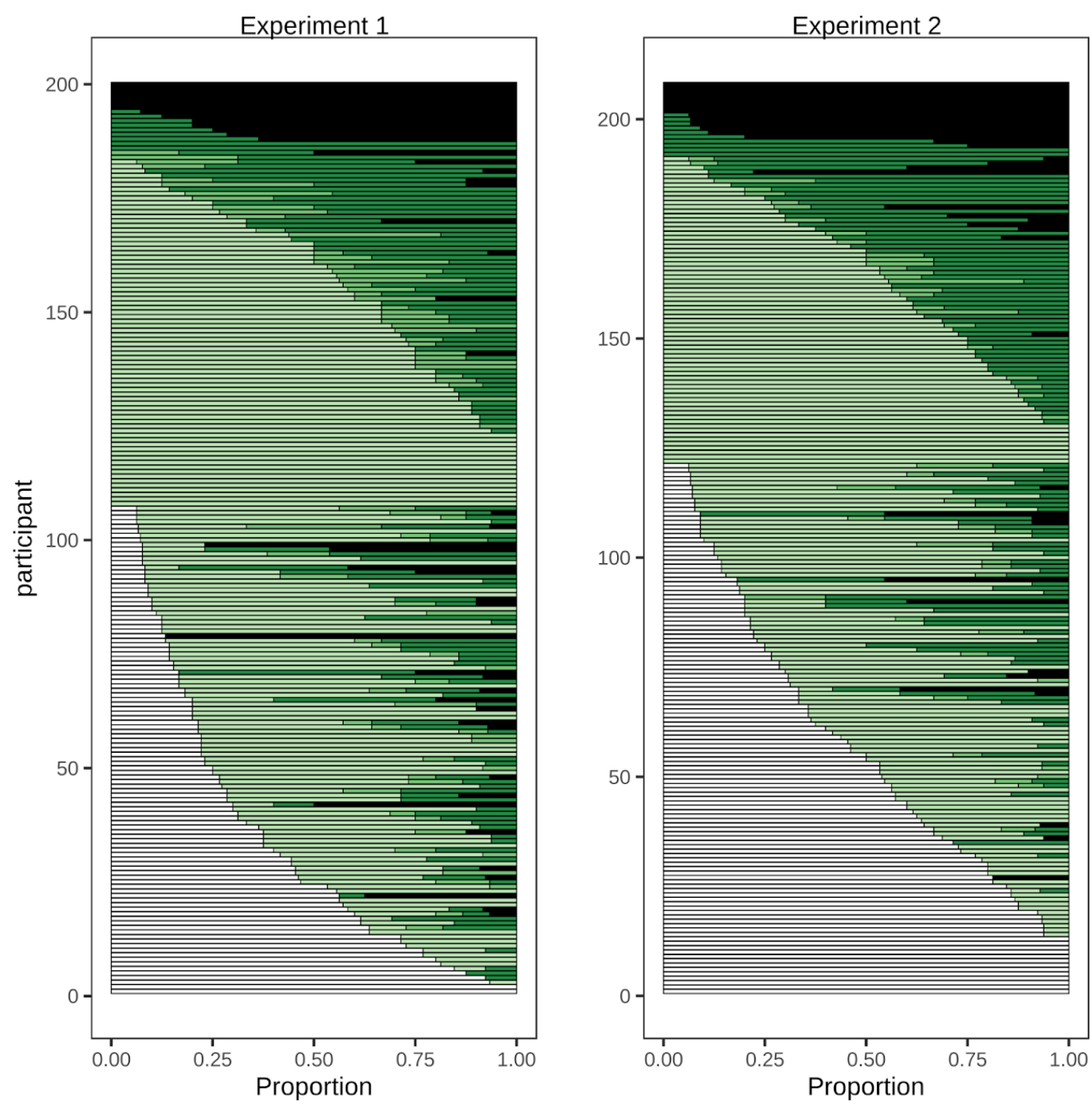

Strategy

$\square \quad$ Stay $\square \quad$ Compromise [s<0.5] $\square \quad$ Compromise [s=0.5] $\square \quad$ Compromise [s>0.5] $\quad \square \quad$ Copy

Proportion of each strategy used by individual participants. Most participants deployed a combination of different strategies throughout the experiment; however, we observe a portion of individuals who always chose to 'Copy' (exp1:6 participants (ppts); exp2: 7 ppts), or always who chose to 'Stay; (exp1: 2 ppts, exp2: 13 ppts). While the overall patter is comparable, in Experiment 2 , the frequency of 'Stay' was higher than Experiment 1 (6\% vs 3\%). 
Fig. S2. Participants' predictions by State in experiment 2.

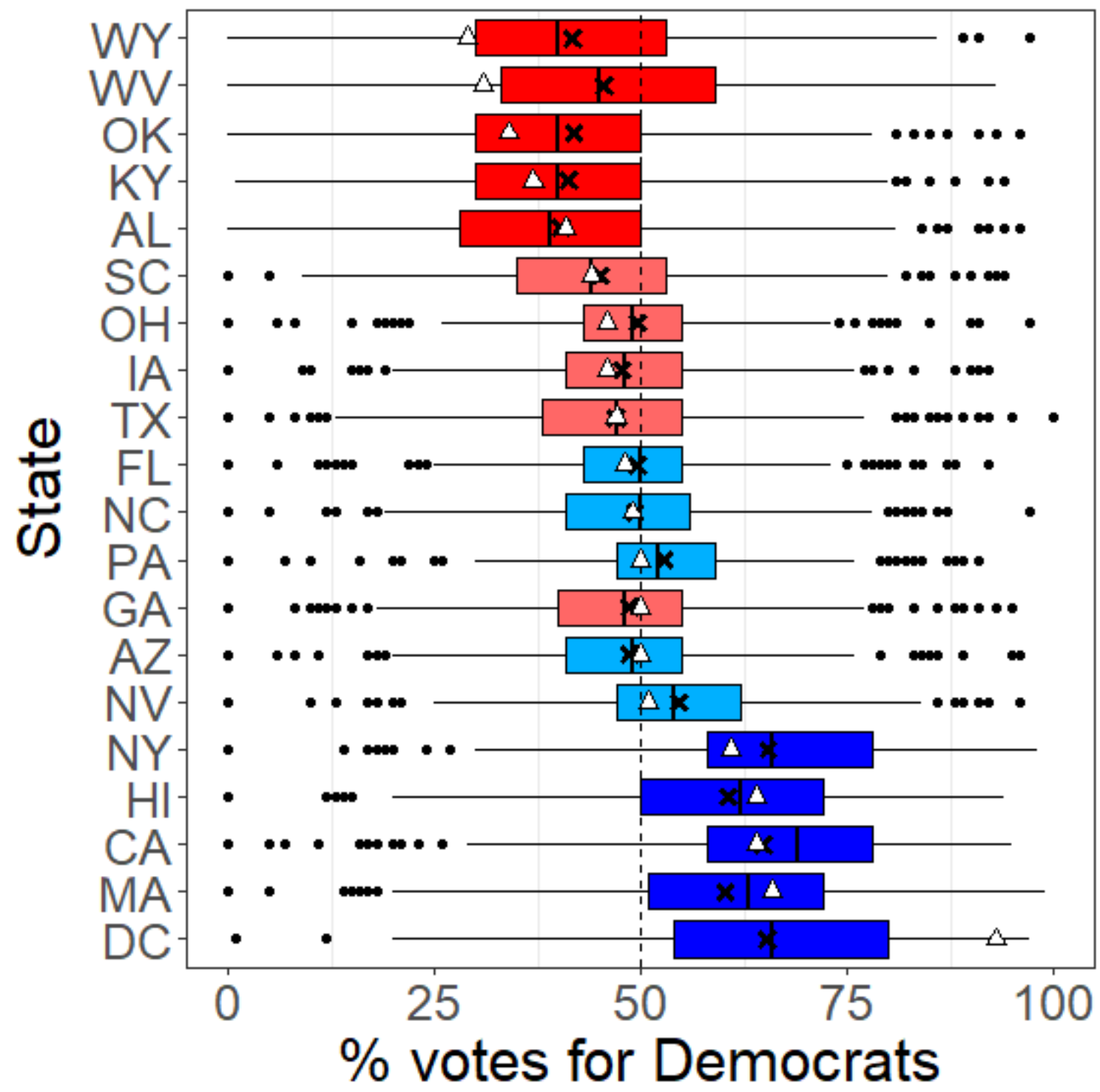

Boxplots represent individual predictions for each State. Black bars represent the median, Black crosses the mean, white filled triangles the real outcome of the elections. Darker colours (ark red; dark blue) indicate states selected in which the poll where less uncertain, while lighter colours (light red; light blue) represent states in which uncertainty was higher. For more information in this distinction, see table S1.

Table S1. States used as stimuli in the Election Task.

\begin{tabular}{|l|l|l|}
\hline State name & Predicted winner & Uncertainty \\
\hline Alabama & Republicans & Low \\
\hline
\end{tabular}




\begin{tabular}{|l|l|l|}
\hline Arizona & Democrats & High \\
\hline California & Democrats & Low \\
\hline Washington D.C. & Democrats & Low \\
\hline Florida & Democrats & High \\
\hline Georgia & Republicans & High \\
\hline Hawaii & Democrats & Low \\
\hline lowa & Republicans & High \\
\hline Kentucky & Republicans & Low \\
\hline Massachusetts & Democrats & Low \\
\hline Nevada & Democrats & High \\
\hline New York & Democrats & Low \\
\hline North Carolina & Democrats & High \\
\hline Ohio & Republicans & High \\
\hline Oklahoma & Republicans & Low \\
\hline Pennsylvania & Democrats & High \\
\hline South Carolina & Republicans & High \\
\hline Texas & Republicans & High \\
\hline West Virginia & Republicans & Low \\
\hline Wyoming & Republicans & Low \\
\hline
\end{tabular}

States were selected to ensure a balance between predicted winners (10 predicted Democrats and 10 predicted Republicans) and uncertainty of the outcome (High or Low), according to polls prediction made by the specialized website http://fivethirtyeight.com. Predictions were consulted in the month before the election (October 2020). 
Table S2. Bayesian Linear Mixed Model Results from Experiment 1

\begin{tabular}{l|cccc} 
& Estimate $(\mathbf{C l})$ & Rhat & Bulk_ESS & Tail_ESS \\
\hline Intercept & $.37(.33, .42)$ & 1.00 & 497 & 1151 \\
$\begin{array}{l}\text { Confidence self: } \\
\text { High }\end{array}$ & $-.05,(-.08,-.01)$ & 1.00 & 2352 & 3228 \\
$\begin{array}{l}\text { Confidence other: } \\
\text { High }\end{array}$ & $.12(.10, .15)$ & 1.00 & 4064 & 4217 \\
$\begin{array}{l}\text { Confidence Self X } \\
\text { Confidence Other }\end{array}$ & $-.07(-.11,-.04)$ & 1.00 & 3696 & 4168
\end{tabular}

Estimates of Bayesian Linear Mixed Model with participants as random intercepts fitted to individual rounds adjustments (s). Values between parentheses indicate $95 \%$ credible intervals. 
Table S3. Bayesian Linear Mixed Model Results from Experiment 2 (including robustness checks)

\begin{tabular}{|c|c|c|c|c|}
\hline & Estimate $(\mathrm{Cl})$ & Rhat & Bulk_ESS & Tail_ESS \\
\hline Intercept & $.32(.20, .43)$ & 1.00 & 591 & 1131 \\
\hline Confidence self: High & $-.03,(-.05,-.00)$ & 1.00 & 5466 & 4239 \\
\hline $\begin{array}{l}\text { Confidence other: } \\
\text { High }\end{array}$ & $.08(.06, .10)$ & 1.00 & 5083 & 4384 \\
\hline $\begin{array}{l}\text { Confidence Self X } \\
\text { Confidence Other }\end{array}$ & $-.03(-.06,-.00)$ & 1.00 & 5048 & 4739 \\
\hline Robustness checks & & & & \\
\hline $\begin{array}{l}\text { Participant's } \\
\text { expertise }\end{array}$ & $-.02(-.19, .15)$ & 1.01 & 595 & 1059 \\
\hline $\begin{array}{l}\text { Desirable outcome: } \\
\text { yes }\end{array}$ & $.01(-.01, .02)$ & 1.00 & 8946 & 4365 \\
\hline Same majority: yes & $.03(.01, .04)$ & 1.00 & 8645 & 4466 \\
\hline Population size & $-.01(-.02,0.00)$ & 1.00 & 5048 & 3935 \\
\hline
\end{tabular}

Estimates of Bayesian Linear Mixed Model with participants as random intercepts fitted to individual rounds adjustments (s). Robustness checks were included to test whether participants would be influenced by seeing: 1) social information in line with their political preferences (Desirable outcome, i.e. a Democrat seeing a prediction of Democrats winning for that state); 2 ) social information showed the same winning party, even if the exact percentages differed (Same majority), and 3) states with larger population sizes, as a proxy for states covered more by the press, and thus easier to predict. Including the robustness checks did not change the effects of confidence (both of self and others) on social information use. Values between parentheses indicate $95 \%$ credible intervals. 
Table S4. Conditional probabilities of adjustment strategies in experiment $1 \& 2$, without random intercepts.

\begin{tabular}{|c|c|c|c|c|c|c|}
\hline \multirow[b]{2}{*}{ Heuristic } & \multicolumn{3}{|c|}{ Experiment 1} & \multicolumn{3}{|c|}{ Experiment 2} \\
\hline & Stay & Compromise & Copy & Stay & Compromise & Copy \\
\hline Treatment & & & & & & \\
\hline LL & $\begin{array}{c}.22 \\
(.18, .27)\end{array}$ & $\begin{array}{c}.76 \\
(.71, .80)\end{array}$ & $\begin{array}{c}.02 \\
(.01, .03)\end{array}$ & $\begin{array}{c}.32 \\
(.29, .36)\end{array}$ & $\begin{array}{c}.61 \\
(.57, .65)\end{array}$ & $\begin{array}{c}.07 \\
(.05, .09)\end{array}$ \\
\hline HL & $\begin{array}{c}.09 \\
(.07, .13)\end{array}$ & $\begin{array}{c}.86 \\
(.82, .89)\end{array}$ & $\begin{array}{c}.04 \\
(.03, .07)\end{array}$ & $\begin{array}{c}.25 \\
(.22, .29)\end{array}$ & $\begin{array}{c}0.71 \\
(.67, .74)\end{array}$ & $\begin{array}{c}.04 \\
(.02, .06)\end{array}$ \\
\hline LH & $\begin{array}{c}.22 \\
(.19, .25)\end{array}$ & $\begin{array}{c}.66 \\
(.62, .69)\end{array}$ & $\begin{array}{c}.12 \\
(.10, .15)\end{array}$ & $\begin{array}{c}.37 \\
(.33, .40)\end{array}$ & $\begin{array}{c}.54 \\
(.51, .58)\end{array}$ & $\begin{array}{c}.09 \\
(.07, .11)\end{array}$ \\
\hline HH & $\begin{array}{c}.18 \\
(.15, .21)\end{array}$ & $\begin{array}{c}.69 \\
(.66 .72)\end{array}$ & $\begin{array}{c}.13 \\
(.11, .16)\end{array}$ & $\begin{array}{c}.27 \\
(.24, .30)\end{array}$ & $\begin{array}{c}.62 \\
(.59, .65)\end{array}$ & $\begin{array}{c}0.11 \\
(.09, .13)\end{array}$ \\
\hline
\end{tabular}

Conditional probabilities of each adjustment strategy (Stay, Compromise, Copy) by condition, estimated by a Bayesian Multinomial Logistic regression. Values between parentheses indicate 95\% credible intervals. Data reported here matches Figure 3 on the main text. 
Table S5. Conditional probabilities of adjustment strategies in experiment $1 \& 2$, with random intercepts

\begin{tabular}{|c|c|c|c|c|c|c|}
\hline \multirow[b]{2}{*}{ Heuristic } & \multicolumn{3}{|c|}{ Experiment 1} & \multicolumn{3}{|c|}{ Experiment 2} \\
\hline & Stay & Compromise & Copy & Stay & Compromise & Copy \\
\hline \multicolumn{7}{|l|}{ Treatment } \\
\hline LL & $\begin{array}{c}.09 \\
(.06, .15)\end{array}$ & $\begin{array}{c}.90 \\
(.85, .94)\end{array}$ & $\begin{array}{c}.00 \\
(.00,00)\end{array}$ & $\begin{array}{c}.19 \\
(.12-28)\end{array}$ & $\begin{array}{c}.80 \\
(.71, .88)\end{array}$ & $\begin{array}{c}.00 \\
(.00,00)\end{array}$ \\
\hline HL & $\begin{array}{c}.02 \\
(.01, .04)\end{array}$ & $\begin{array}{c}.97 \\
(.95, .99)\end{array}$ & $\begin{array}{c}.00 \\
(.00,00)\end{array}$ & $\begin{array}{c}.09 \\
(.5, .14)\end{array}$ & $\begin{array}{c}.90 \\
(.85, .94)\end{array}$ & $\begin{array}{c}.00 \\
(.00,00)\end{array}$ \\
\hline LH & $\begin{array}{c}.15 \\
(.10, .22)\end{array}$ & $\begin{array}{c}.84 \\
(.78, .90)\end{array}$ & $\begin{array}{c}.00 \\
(.00,01)\end{array}$ & $\begin{array}{c}0.23 \\
(.15, .33)\end{array}$ & $\begin{array}{c}.76 \\
(.66, .85)\end{array}$ & $\begin{array}{c}.00 \\
(.00,00)\end{array}$ \\
\hline HH & $\begin{array}{c}.10 \\
(.06, .15)\end{array}$ & $\begin{array}{c}.85 \\
(.85, .93)\end{array}$ & $\begin{array}{c}.00 \\
(.00,01)\end{array}$ & $\begin{array}{c}0.11 \\
(.06, .17)\end{array}$ & $\begin{array}{c}00.89 \\
(.82, .93)\end{array}$ & $\begin{array}{c}.00 \\
(.00,00)\end{array}$ \\
\hline
\end{tabular}

Conditional probabilities of each adjustment strategy (Stay, Compromise, Copy) by condition, estimated by a Bayesian Multinomial Logistic regression with participant ID included as random intercept. Values between parentheses indicate 95\% credible intervals. 
Table S6. Results of regression analysis with confidence as continuous variable in experiment 1

\begin{tabular}{l|cccc} 
& Estimate $(\mathbf{C l})$ & Rhat & Bulk_ESS & Tail_ESS \\
\hline Intercept & $.38(.32, .45)$ & 1.00 & 1810 & 2644 \\
$\begin{array}{l}\text { Confidence self } \\
{[1-10]}\end{array}$ & $-.01,(-.02,-.00)$ & 1.00 & 5046 & 4533 \\
$\begin{array}{l}\text { Confidence other } \\
{[1-10]}\end{array}$ & $.03(.02, .03)$ & 1.00 & 13441 & 4564 \\
$\begin{array}{l}\text { Confidence Self X } \\
\text { Confidence Other }\end{array}$ & $-.00(-.00,-.00)$ & 1.00 & 11186 & 4669
\end{tabular}

Estimates of Bayesian Linear Mixed Model with participants as random intercepts fitted to individual rounds adjustments (s), in experiment 1. Values between parentheses indicate $95 \%$ credible intervals. Contrary to the model reported in the main text, confidence was included as a continuous variable, exploting the full variation present in the confidence scale (range 1-10). The results are qualitatively identical to the model reported in the main text: high confidence of self led to a reduced use of social information, while high confidence of others led to an increase in social information use. Also consistent with the result in the main text, confidence of others had a stronger effect than confidence of self. In the main text we report confidence as a categorical variable to facilitate the comparison with the results of experiment 2 . 